\title{
APPARATUS, SYSTEM, METHOD AND ASSOCIATED TECHNOLOGIES OF IN-LINE VORTEX MIXING OF MINI - GRANULES WITH HOMOGENIZED HEAVY OIL OR RESIN OR BITUMEN, MAINLY IN THE PREPARATION OF THE ASPHALT MIX
}

\author{
Mihai Netid \\ Master Degree \\ Roads, Materials, and Mechanization in Construction \\ Road design engineer
}

\begin{abstract}
Widely used for its convenience, hot mix asphalt, is the most common used flexible pavement all around the world. It is also known as blacktop or bitumen, and sometimes just hot mix. Hot mix pavements are classified mainly as dense-graded mixes, stone matrix mixes, and open-graded hot mix asphalt. There are also other types of asphalt but are limited to maintenance and rehabilitation works.
\end{abstract}

Keywords: in-line vortex, heavy oil, resin, bitumen, homogenization, asphalt mix.

In road construction technologies, the bitumen has a very important role as binder for the asphalt mixture. The viscoelastic, adhesive and with a black or dark brown material is obtained from petroleum distillation.

Bitumen is a viscoelastic material, adhesive and appears mostly black. It can be obtained as a residue from petroleum distillation and can be further processed by air blowing process to alter its physical properties. Natural bitumen, such as the occurrence in natural asphalt lakes, differs from industrial produced bitumen. They are unrefined and often contain more light compounds, mineral particles and occasionally water.

Bitumen as a material, can be divided into four fractions within two steps. The first step is the precipitation of asphaltenes, done by filtering bitumen dissolved in $\mathrm{n}$-heptane. The remaining part, called maltenes, is then in the second step separated by liquid column chromatography into saturates, aromatics and resins. Polarity increases form saturates to resins and asphaltenes are in the polarity range of aromatics and resins. 
Asphaltenes form a black powder and decompose when heated above $350^{\circ} \mathrm{C}$. The asphaltene fraction consists of large molecules containing hetero atoms like oxygen, nitrogen, sulfur and metal atoms. Asphaltenes have a major contribution on all physical properties, as they increase alongside of resins. During ageing bitumen gets less viscous, so their fluorescence signal decreases.

Resins are a black solid at room temperature and liquefy at higher temperature. The exhibited fluorescence is contributed to its molecular structure containing aromatic rings. They increase during ageing.

Aromatics are viscous and appear dark red. They contribute the most to the fluorescence of bitumen. Other fractions (most resins) seem to quench the fluorescence of the aromatics leading to the spectrum of maltenes. Aromatics act as solution for asphaltenes and resins and are decreasing during ageing.

Saturates are a colorless liquid, which behaves purely viscous like aromatics. The saturate concentration is not altered during ageing, so their contribution to any property remains constant.

During ageing the fractions asphaltenes and resins increase in content as aromatics decrease. Due to the change in these three fractions the fluorescence signal lessens. Infrared spectroscopy indicates that oxygen is taken up during ageing - oxidation process.

\section{Description of the apparatus}






Figure 1. An example of a 3-D model of the apparatus with transparent housing and internal parts.

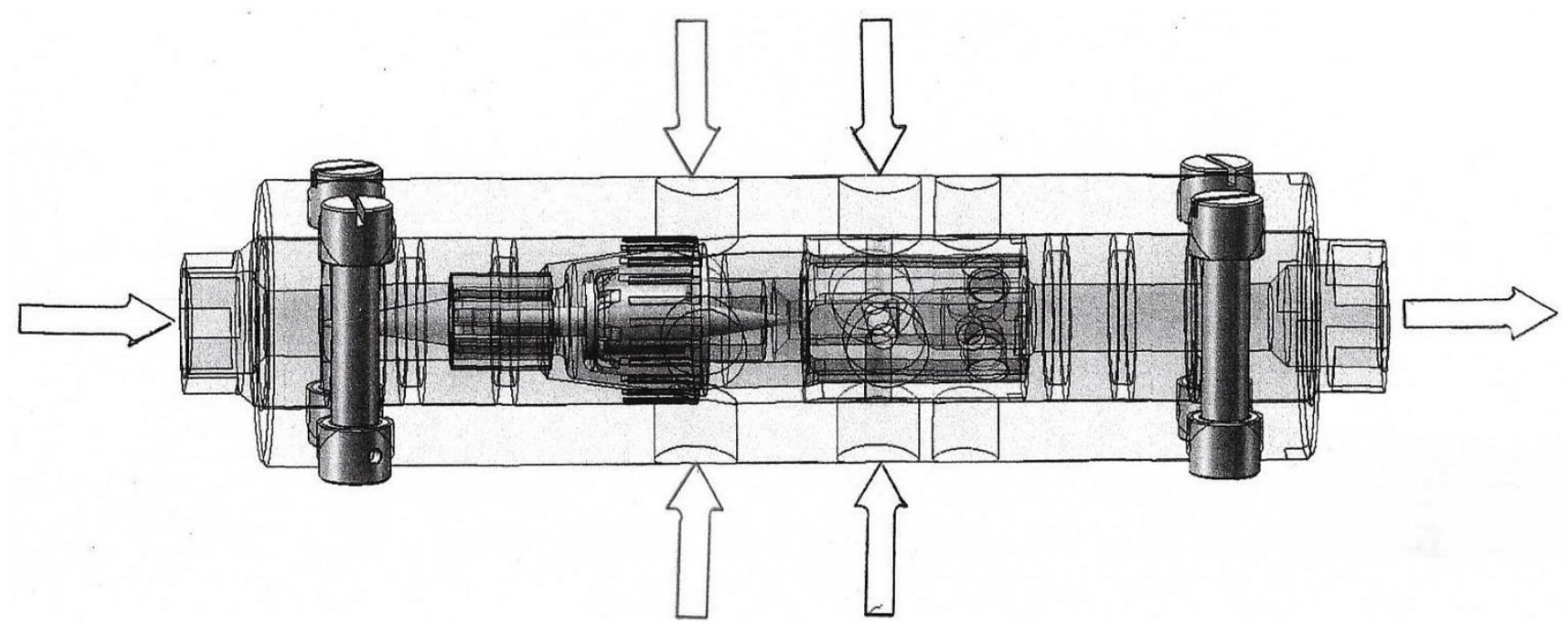

Figure 2. An example of a 3-D model of the homogenization apparatus with transparent housing and internal parts and indication of all inputs and output. 


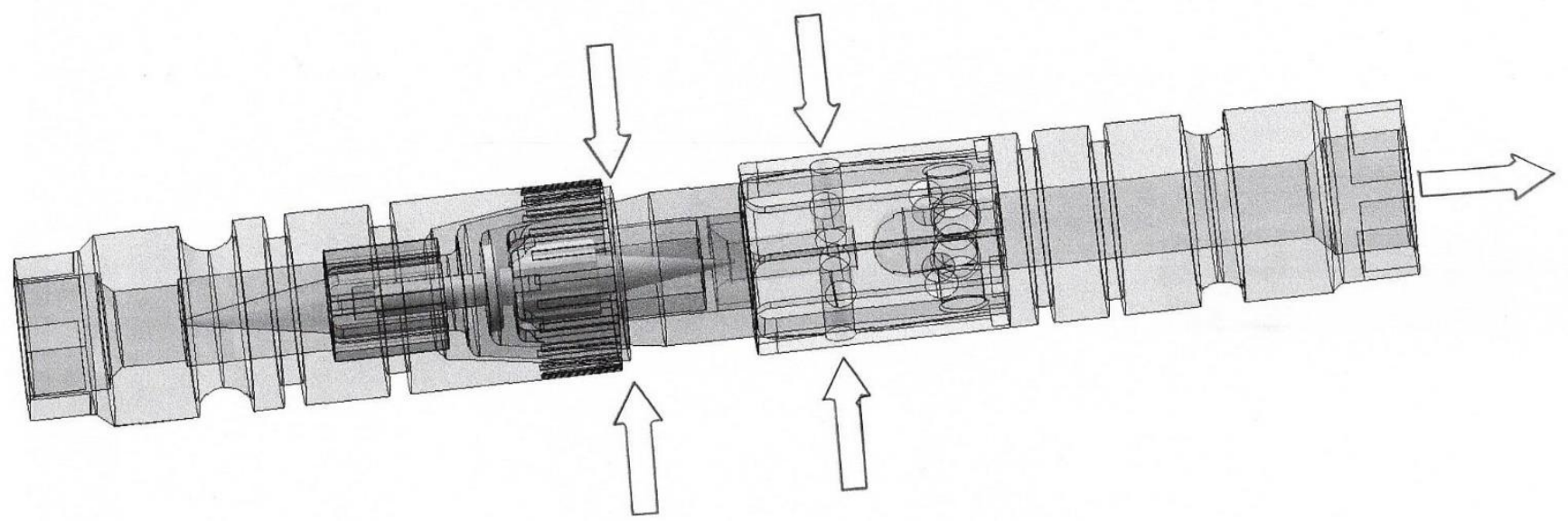

Figure 3. An example of a 3-D model of the homogenization apparatus with transparent housing and internal parts and indication of all inputs and output


Figure 4 is an example of a 3-D models of the internal parts groups of the apparatus 


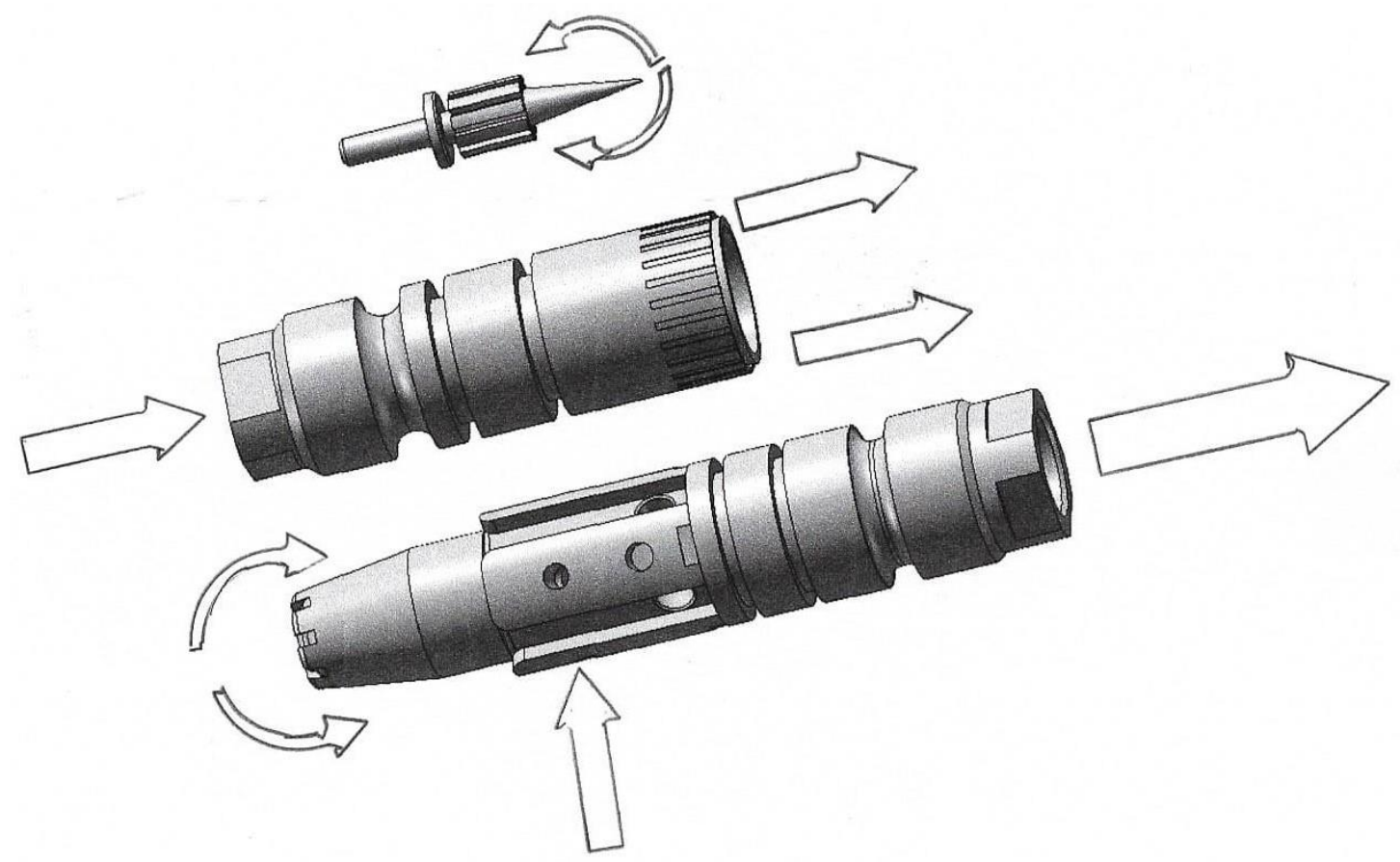

Figure 5. An example of a 3-D model of the internal parts groups of the apparatus

Various embodiments of the invention allow the next advantage:

It will be appreciated by those skilled in the art that various omissions, additions and modifications may be made to the methods and structures described above without departing from the scope of the invention. All such modifications and changes are intended to fall within the scope of the invention, as defined by the appended claims.

\section{In conclusion}

The apparatus for in-line stabilization of the hydrodynamic fluids conditions, including the aging effect, containing interconnected systems conversion and optimization of hydrodynamic and turbulent flow parameters - a coherent system of step a smooth transformation of the form of flow and smooth distribution of parts flow between the system of coaxial tapered channel direction of each conical channel large cone base toward the direction of flow, with the ratio of the cross-areas section of the channels remains constant over the entire channel length, and typically the crosssectional outer channel area exceeds the cross-sectional internal area of the channel, at 
least 2 times, and the volume of the annular channel downstream of the exit from the coaxial channels exceeds the capacity of coaxial tapered channels as at least 100 times

The invention relates to a fluid composite, a device for producing the fluid composite, and a system for producing an aerated fluid composite therewith, and more specifically a fluid composite made of a fuel and its oxidant for burning as part of different systems such as fuel burners or combustion chambers and the like. The invention also relates to an emulsion, an apparatus for producing an emulsion, a system for producing an emulsion with the apparatus for producing the emulsion, a method for producing a dynamic preparation with the emulsion, and more specifically to a new type of a stable liquid/liquid emulsion in the field of colloidal chemistry, such as a water/fuel or fuel/fuel emulsion for all spheres of industry.

\section{References}

1. https://www.thebalancesmb.com/hot-mix-asphalt-types-844575.

2. https://www.researchgate.net/publication/306395170_Investigation_of_Aged_No n-aged_Bitumen_and_their_Bitumen_Fractions.

3. Simanzhenkov V, Idem R. Crude Oil Chemistry. Marcel Dekker, Inc.; 2003.

4. Asphalt Institute, Eurobitume. The Bitumen Industry - A Global Perspective Production, chemistry, use, specification and occupational exposure. second Edition. Asphalt Institute Inc. and European Bitumen Association-Eurobitume; 2011.

5. Lesueur D. The colloidal structure of bitumen: Consequences on the rheology and on the mechanisms of bitumen modification. Adv Colloid Interface Sci 2009;145:42-82. 